\title{
Evaluation of cultures of Saccharomyces cerevisae as baits for Triatoma dimidiata and Triatoma pallidipennis
}

\author{
Flávio E Pimenta, Liléia Diotaiuti, Anna C Lustosa Lima, Marcelo G Lorenzo ${ }^{+}$
}

Laboratório de Triatomíneos e Epidemiologia da Doença de Chagas, Centro de Pesquisa René Rachou-Fiocruz, Av. Augusto de Lima 1715, 30190-002 Belo Horizonte, MG, Brasil

We tested the attraction of Triatoma dimidiata and T. pallidipennis to traps baited with yeast volatiles. Two traps were simultaneously presented in opposite sides of an experimental arena. One trap presented a yeast culture in sucrose solution, while the other contained sucrose solution as control. A first experimental series was done without offering a central refuge for bugs. In a second series, one shelter where the insects could hide was offered and the traps were presented afterwards. In the first series, yeast baited traps attracted significantly more insects than control ones for both species. In the second series, T. pallidipennis was significantly attracted to yeast, whereas T. dimidiata was not attracted. The potential use of yeast baited traps for capturing these vectors of Chagas disease is discussed.

Key words: triatomines - traps - control

Triatoma dimidiata is considered the second most important vector of Chagas disease in Central America and North-Western South America. The control of T. dimidiata is more difficult than that of highly domiciliated species, like T. infestans or Rhodnius prolixus, due to the fact that it is found in sylvatic as well as in peridomestic and domestic environments. T. pallidipennis is another relevant triatomine present in part of Mexico that has a significant potential as vector of Trypanosoma cruzi over its distribution range (Espinoza-Gomez et al. 2002, Enger et al. 2004). The capture of sylvatic triatomines is difficult due to their sparse presence in relatively diverse ecotopes that frequently do not offer open access for sampling purposes. Different methods have been used, including light traps (Noireau \& Dujardin 2001, Vazquez-Prokopec et al. 2004), devices baited with live hosts (Noireau et al. 2002) or manual search. Nevertheless, these can be either limited due to their capacity of capturing exclusively adults or time limited due to the need of host maintenance.

This work analyses the potential use of scents that apparently mimic host odours for the attraction and capture of these insects. The chemical ecology of vectors of Chagas disease deserves attention, due to its potential for supporting the development of detection and control tools. Guerenstein et al. (1995) reported an attractive effect of yeast cultures in T. infestans, probably due to their emission of $\mathrm{CO}_{2}$ and other odours emitted by hosts. This work aims to test whether yeast cultures can be used as baits for traps to attract and capture $T$. dimidiata and T. pallidipennis.

Financial support: UNDP/World Bank/WHO Special Programme for Research and Training in Tropical Diseases, Fiocruz, ProbicFapemig

+Corresponding author: marcelo@ cpqrr.fiocruz.br

Received 11 September 2006

Accepted 26 February 2007
The insects used were reared at our laboratory and fed with mice and pigeon blood. We used 400 third and fourth instar larvae of $T$. dimidiata and 252 fourth instar larvae of $T$. pallidipennis for the experiments. The experimental insects were starved for approximately 20 days after ecdysis and kept under a natural illumination cycle. The temperature of the experimental room was $25 \pm 2{ }^{\circ} \mathrm{C}$. The assays were done in square experimental arenas with a side length of $100 \mathrm{~cm}$ and a height of 20 $\mathrm{cm}$. The arenas had their substrate completely covered with Kraft paper that was changed after each assay. The insects were released in the centre of the arena using an inverted recipient that could be raised from a distant point by means of a thread. In the assays, two traps of the model designed by Guerenstein et al. (1995) were presented simultaneously at opposite sides of the arena (see Figure). One trap presented a flask with a yeast culture in sucrose solution prepared as in Guerenstein et al. (1995), while the other only had the sucrose solution in an identical flask as a control stimulus. The insects were released in the late afternoon and the number of insects captured at each trap was counted in the following morning $4 \mathrm{~h}$ after dawn.

Two series of assays were accomplished: (1) assays at which no refuge was offered to the insects inside the arena (T. dimidiata 148 larvae, 5 assays; $T$. pallidipennis 180 larvae, 6 assays) and (2) assays with a refuge offered to the bugs ( $T$. dimidiata 252 larvae, 8 assays; $T$. pallidipennis 72 larvae, 6 assays). In this second series, we firstly offered an artificial refuge placed in the centre of the arena that was mounted using a piece of corrugated cardboard of $10 \mathrm{~cm} \times 20 \mathrm{~cm}$ bent in its middle line, creating a shelter of $10 \mathrm{~cm} \times 10 \mathrm{~cm}$ with two lateral entrances (Lorenzo \& Lazzari 1996). Assays did not initiate until all insects had entered the shelter, therefore, in that series the insects spent more than one day in the arena until the assay started. Using this experimental design, we tested the capacity of yeast baits for activating each species out of the shelter. With the purpose of evaluating the attractive effect of the volatiles 


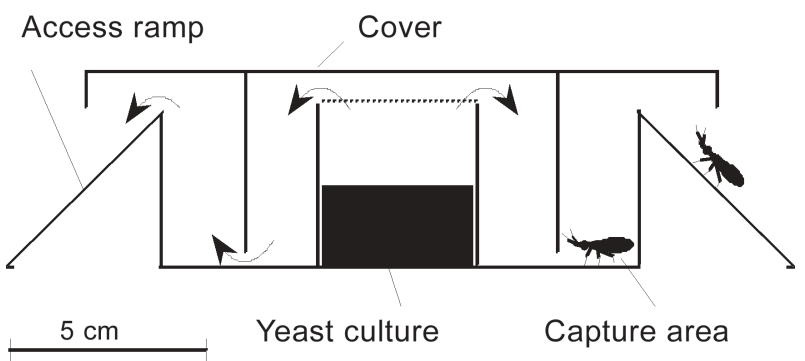

Trap prototype used for testing the attractivity of yeast culture volatiles for Triatoma dimidiata and T. pallidipennis.

released by yeast cultures, the number of individuals captured in yeast baited traps, expressed as a proportion in relation to the total capture of both traps, was compared by a test for one proportion using as null hypothesis an expected proportion of 0.5 , with a $95 \%$ confidence level. The Kruskal-Wallis test was used to evaluate the effects of treatment, i.e., a refuge offered or not, and species on the capture performance of both traps. For a posteriori multiple comparisons between series, we used Mann-Whitney tests with their significance level adjusted by the Bonferroni method $(\alpha=0.0125)$.

The results of the first series of experiments indicated that this trap design is capable of capturing both species of bugs. In the assays with T. pallidipennis, the statistical analysis revealed that the proportion of insects captured by yeast baited traps was significantly larger than in control ones (Table, test for one proportion, $\mathrm{n}=149, \mathrm{p}<0.0001$ ). Yeast baited traps also captured a higher proportion of T. dimidiata bugs than control ones (Table, test for one proportion, $\mathrm{n}=53, \mathrm{p}<0.0001$ ).

When the assays offering refuges were performed with T. pallidipennis, only yeast baited traps captured insects (Table, test for one proportion, $\mathrm{n}=25, \mathrm{p}<0.0001$ ). Nevertheless, in a similar series with $T$. dimidiata, the comparison among the capture of yeast baited and control traps did not show a significant difference (Table, test for one proportion, $\mathrm{n}=8, \mathrm{p}=0.289$ ).

The comparison of the total capture obtained in the different series indicated an effect of the treatment, refuge offered or not, and the species tested (Kruskal-Wallis test, $\mathrm{df}=3, \mathrm{p}=0.0002$ ). The statistical analysis showed that $T$. pallidipennis responds to yeast baits in a different manner whether a refuge is offered or not (MannWhitney test, 6 vs 6 replicates, $p=0.0043$ ), indicating that the presence of a shelter lowers the capture efficiency of traps. On the other hand, when a shelter was offered to this species only yeast baited traps captured bugs, suggesting that resting bugs motivated for host searching can be driven out of refuges by yeast volatiles. When T. pallidipennis and T. dimidiata were tested without shelters the former was more activated by yeast than the latter (Mann-Whitney test, 6 vs 5 replicates, $\mathrm{p}=0.0043$ ). A comparison between $T$. dimidiata series shows that it responds to yeast volatiles in a dramatically different manner whether a refuge is offered or not (Mann-Whitney test, 8 vs 5 replicates, $\mathrm{p}=0.0016$ ), indicating that the presence of a shelter almost nullifies the capture efficiency of traps. The comparison of experimental series performed offering refuges for both species also showed that $T$. dimidiata was sensibly less activated and therefore, it could not be driven out of refuges using yeast volatiles (Mann-Whitney test, 8 vs 6 replicates, $\mathrm{p}=0.0013$ ).

Yeast baited traps were shown to attract and capture several species of triatomine bugs (Guerenstein et al. 1995, Lorenzo et al. 1999, Pires et al. 2000). In this report, we have demonstrated that $T$. dimidiata is attracted by yeast baited traps, even though this attraction is weak when compared with that observed for $T$. infestans or $R$. prolixus. On the other hand, T. pallidipennis was strongly attracted by yeast baits and easily captured by these traps. Moreover, insects of this species could apparently be drawn out from refuges by these odours, suggesting that this bait is capable of activating resting insects and subsequently attract them to traps.

The results obtained when refuges were offered indicate that this bait is not strong enough to drive resting T. dimidiata out from a shelter. Then, yeast baited traps would work capturing mostly wandering specimens of this species. Therefore, a complementary stimulus or even an alternative bait may be necessary for attracting resting $T$. dimidiata.

Most of the studies analyzing the orientation to hosts in triatomine bugs were developed with T. infestans (Taneja \& Guerin 1995, Guerenstein \& Guerin 2001, Barrozo \& Lazzari 2004a, b) and do not allow to consider that similar results are to be obtained if the same experiments were done with other vector species. No previous reports have presented capture data for traps using odorant baits for triatomines. The only other traps used to date for capturing all stages of triatomines are those using live hosts as baits (Noireau et al. 2002) that require host care and can therefore be limited depending on field work conditions. The characterization of the role of odours in the orientation of triatomine bugs to their hosts is consequently fundamental for the improvement of existing detection techniques or the development of new detection tools. The perspectives

TABLE

Total number of assayed insects, total capture and attraction of insects of both species in the different experimental series

\begin{tabular}{lcrc}
\hline & Total capture & Attraction & Total number \\
\hline Triatoma pallidipennis (no refuge) & 149 & 130 & $180(6)$ \\
T. pallidipennis (refuge) & 25 & 25 & $72(6)$ \\
T. dimidiata (no refuge) & 53 & 40 & $148(5)$ \\
T. dimidiata (refuge) & 8 & 6 & $252(8)$ \\
\hline
\end{tabular}

Total capture: number of test insects that were captured by both traps (control and yeast baited); attraction: number of captured insects that were caught by yeast baited traps; total number: insects tested in each series (number of assays). 
for using yeast baits in the field will depend on new reports testing them under such conditions in the countries where these species transmit T. cruzi to humans. A relevant conclusion driven from current data is that host odours can promote the capture of these bugs in pit traps. Moreover, pit traps have showed successful for the capture of several triatomine species and should be considered for use in association with any new host related attractants (Guerenstein et al. 1995, Lorenzo et al. 1999, Pires et al. 2000).

\section{REFERENCES}

Barrozo RB, Lazzari CR 2004a. The response of the blood-sucking bug Triatoma infestans to carbon dioxide and other host odours. Chem Senses 29: 319-329.

Barrozo RB, Lazzari CR 2004b. Orientation behaviour of the blood sucking bug Triatoma infestans to short-chain fatty acids: synergistic effect of L-lactic acid and carbon dioxide. Chem Senses 29: 833-841.

Enger KS, Ordonez R, Wilson ML, Ramsey JM 2004. Evaluation of risk factors for rural infestation by Triatoma pallidipennis (Hemiptera: Triatominae), a Mexican vector of Chagas disease. J Med Entomol 41: 760-767.

Espinoza-Gomez F, Maldonado-Rodriguez A, Coll-Cardenas R, Hernandez-Suarez CM, Fernandez-Salas I 2002. Presence of Triatominae (Hemiptera, Reduviidae) and risk of transmission of Chagas disease in Colima, Mexico. Mem Inst Oswaldo Cruz 97: 25-30.

Guerenstein PG, Guerin P 2001. Olfactory and behavioural responses of the blood-sucking bug Triatoma infestans to odours of vertebrate hosts. J Exp Biol 204: 585-597.
Guerenstein PG, Lorenzo MG, Núñez JA, Lazzari CR 1995. Baker's yeast, an attractant for baiting traps for Chagas' disease vectors. Experientia 51: 834-837.

Lorenzo MG, Lazzari CR 1996. The spatial pattern of defecation in Triatoma infestans and the role of faeces as a chemical mark of the refuge. J Insect Physiol 42: 903-907.

Lorenzo MG, Manrique G, Pires HHR, Brito Sánchez MG, Diotaiuti L, Lazzari CR 1999. Yeast culture volatiles as attractants for Rhodnius prolixus: electroantennogram responses and captures in yeast-baited traps. Acta Trop 72: 119-124.

Noireau F, Dujardin JP 2001. Flight and nutritional status of sylvatic Triatoma sordida and Triatoma guasayana. Mem Inst Oswaldo Cruz 96: 385-389.

Noireau F, Abad-Franch F, Valente SAS, Dias-Lima A, Lopes CM, Cunha V, Valente VC, Palomeque FS, de Carvalho Pinto CJ, Sherlock I, Aguilar M, Steindel M, Grisard EC, Jurberg J 2002. Trapping Triatominae in silvatic habitats. Mem Inst Oswaldo Cruz 97: 61-63.

Pires HHR, Lorenzo MG, Lazzari CR, Diotaiuti L 2000. Performance of yeast-baited traps with Triatoma sordida, Triatoma brasiliensis, Triatoma pseudomaculata, and Panstrongylus megistus in laboratory assays. Rev Panam Salud Públical Pan Am J Public Health 7: 384-388.

Taneja J, Guerin PM 1995. Oriented responses of the triatomine bugs Rhodnius prolixus and Triatoma infestans to vertebrate odours on a servosphere. J Comp Physiol A 176: 455-464.

Vazquez-Prokopec GM, Ceballos LA, Kitron U, Gurtler RE 2004. Active dispersal of natural populations of Triatoma infestans (Hemiptera: Reduviidae) in rural Northwestern Argentina. J Med Entomol 41: 614-621. 